\title{
Computation of Reactive Power in the Framework of the New Spanish Grid Code using Test Fields of Wind Turbines Submitted to Voltage Dips
}

\author{
E. Gómez, J. A. Fuentes, A. Molina, F. Ruz, M. Cañas, F. Jiménez
}

\begin{abstract}
This paper is focused on the computation of the reactive power generated by wind turbines when they are submitted to a voltage dip in the framework of the new Grid Codes. Taking advantage of the voltage dips field tests carried out to the Gamesa G80 wind turbines, several definitions of reactive power are calculated and the results obtained for a representative three-phase voltage dip test are presented.
\end{abstract}

Index Terms - Voltage dips, wind turbines, reactive power, grid codes

\section{INTRODUCTION}

Wind power has become a competitive technology for clean energy production in recent years. The developments of induction generators and power electronics have contributed to achieve this state. This is a consequence of the policies applied in some countries, mainly in Europe as shown in Table I. Due to these policies, renewable sources of energy, which can be considered as national resources, have and will play an important role in reducing the energy imports. Integration of Distributed Generation in the utility grid offers technical, environmental and economical benefits, from both the utility and the end-user point of view, [1] [2] [3].

In Europe, Germany heads the list with 18428 MW connected to the electrical network, followed by Spain $10027 \mathrm{MW}$ at the end of 2005, $2119 \mathrm{MW}$ at the end of 2006 - and Denmark - 3122 MW - These three countries alone accounted for $79 \%$ of the wind power capacity installed in the European Union by the end of 2005, Table I. USA is the third country in the world with an installed capacity of wind power of $10492 \mathrm{MW}$.

Therefore, it is evident that nowadays and in the future, it may not be quite so straigthforward the connection of wind farms to the grid, whereas the earlier small wind farms could be connected to the existing grids - without specific restrictive requirements - Among other reasons, this is due to the impact that wind turbines may have on the slow voltage variations, flicker, harmonics and voltage dips. Voltage dips and their effects on industrial installations have been widely studied, [4].

E. Gómez and M. Cañas are with the Renewable Energy Research Institute and the Dept. of Electrical, Electronic, and Control Eng, EPSA. Universidad de Castilla-La Mancha, 02071 Albacete (SPAIN); emilio.gomez@uclm.es

J. A. Fuentes, A. Molina and F. Ruz are with the Department of Electrical Eng. Universidad Politécnica de Cartagena, 30202 Cartagena (SPAIN); juanalvaro.fuentes@upct.es

F. Jiménez is with Engineering Department, Gamesa Eólica S.A., Polígono Agustinos, calle A, E-31013 Pamplona (SPAIN); fjimenez@eolica.gamesa.es
TABLE I

EUROPE WIND ENERGY GENERATING CAPACITY BY END OF 2005

\begin{tabular}{|c|c|}
\hline Country & Capacity(MW) \\
\hline Germany & 18.428 \\
\hline Spain & 10.027 \\
\hline Denmark & 3.122 \\
\hline Italy & 1.717 \\
\hline UK & 1.353 \\
\hline Netherlands & 1.219 \\
\hline Portugal & 1.022 \\
\hline Austria & 819 \\
\hline France & 757 \\
\hline Greece & 573 \\
\hline Sweden & 500 \\
\hline Ireland & 495 \\
\hline Belgium & 167 \\
\hline Finland & 82 \\
\hline Poland & 73 \\
\hline Luxembourg & 35 \\
\hline Estonia & 30 \\
\hline Czech Republic & 26 \\
\hline Latvia & 26 \\
\hline Hungary & 17 \\
\hline Lithuania & 7 \\
\hline Slovakia & 5 \\
\hline$E U-25$ total & 40.504 \\
\hline Accesion Countries & 28 \\
\hline EFTA Countries & 279 \\
\hline Other Countries & 86 \\
\hline
\end{tabular}

Source: European Wind Energy Association (EWEA)

As the wind farm penetration in power systems increases, many national grid codes are demanding additional requirements to wind power installations in order to integrate them with other conventional types of generation. Specifically, the national grid codes require uninterrupted generation throughout power system disturbances supporting the network voltage and frequency, therefore, extending characteristics such as low voltage ride through, or reactive and active power capabilities. Low voltage ride through is particularly important to maintain voltage stability, especially in areas with high concentration of wind power generation -wind turbines were disconnected from the grid when the terminal voltage fell below $80 \%$ according to the previous practice, [5]—. Premature tripping of wind generators due to power system disturbances may put in risk the stability of the system, wich contributes to the amplification of the disturbance, [6], [7].

Among the power system disturbances, the disturbances 
involved in low voltage ride through studies, [7], are voltage dips mainly due to short-circuits and earth faults in the grid. A voltage dip is a sudden reduction (between $10 \%$ and $90 \%$ ) of the voltage at a point within the electrical system, which lasts for half a cycle to $1 \mathrm{~min}$, [8]. These faults in the power system, even far away from the location of the wind farm, can generate a voltage dip at the connection point of the wind turbines.

Regarding wind turbines, and attending to the formerly exposed, it is evident that the most sensible component to voltage dips is the power electronic converter. This is usually a Voltage Source Converter (VSC) with current control based on IGBTs, which are controlled by means of Pulse Width Modulation (PWM) techniques with high switching frequencies to achieve high power quality. The main drawback in using VSC converters is their sensitivity to voltage disturbances, such as voltage dips. In general, for this type of converter, a sudden decrease in the grid voltage usually causes an increase in their current, as the control attempts to maintain the power of the DC link constant. This can lead to tripping of the converter to prevent overcurrents, in order to protect the IGBTs. Moreover, most faults are unbalanced and generate as a result unbalanced dips, which produce undesirable power oscillations of low order frequencies, which in turn result in current harmonics and poor DC link voltage regulation. As a consequence, maintaining an adequate functioning of the power electronic converter of the wind turbines in the presence of voltage dips can be considered as one of the main objectives when assuring a correct operating of these systems, by virtue of the regulation previously quoted.

The recently published REE grid code, specifies that the wind farm must support voltage dips at the point of interconnection with the transmission network, without tripping.

\section{Voltage Dips Literature ReView}

In the last years, most of the TSOs (Transmission System Operators) with a great percentage of renewable origin power plants have made diverse modifications in their Grid Codes to integrate these facilities (like on-shore and off-shore wind farms). Thus, additional requirements have been imposed to mantain suitable levels of operation in the transport and distribution grid. Due to the great advance that has been carried out in the last decade in the design and development of new devices of power electronics, its use in diverse installations connected to the grid has been standardised. The weak point of these devices is their sensitivity to small disturbances in the voltage waveform like amplitude and frequency variations.

The disturbances in the voltage waveform can be caused by a number of parameters such as the start-up of a large induction motor or the correct clearance of faults. Among the power system disturbances, voltage dips mainly due to shortcircuits and earth faults in the grid, [7], are the disturbances involved in low voltage ride through studies. Regarding the power system disturbances, voltage dips mainly due to shortcircuits and earth faults in the grid, [7], are the disturbances involved in low voltage ride through studies. The vast implantation of wind energy in certain European countries has caused a great development wind energy-related technologies.
As mentioned before, the devices based on power electronics are sensible to disturbances such as voltage dips. Consequently, a dip originated by the correct elimination of a fault in the grid causes the shutdown of a wind turbine due to the firing of the over-current protections in the rotor of the wind turbine generator.

Basically, the new Grid Codes impose certain procedures in the wind farms certain procedures to fulfil in case of voltage dips occur. They may be summarized in two steps: firstly, wind farms cannot be disconnected in the presence of voltage dips, and second, wind farms can be disconnected of the grid in case of severe voltage dips, thougth they must be reconnected as soon as possible to help the TSO in the recovery of the fault.

The fault that originates the dip may occur far away from the wind farm, and sometimes the type of voltage dip observed by the wind turbine may differ from the original, [9]. The detection and classification of voltage dips is a very interesting subject, being widely studied, [10], [11]. The necessity to fulfil the new Grid Code has impelled the development of new designs of devices of power electronic devices for their application in wind turbines to keep them connected to the network during a voltage dip. A clear example is the active crowbar. The main problem of these devices is that they sometimes do not differentiate between a voltage dip and a severe fault. For the first case, the wind turbine should remain connected to the grid and in the second the safety measures should be activate to safeguard the integrity of the devices of the wind turbine. To minimise this problem, techniques based in Voltage Source Converters, [12], and Pulse Width Modulation, [13], have been developed.

In economic terms the effect of voltage dips is very high [14], estimating utilities these costs normally under the level, they really are. Voltage dips and partial interruptions have proved to be the most costly power quality variation to the consumer.

\section{REACTIVE POWER DEFINITIONS}

The different studied reactive power definitions are described in the following subsections.

\section{A. D1 definition}

This method was introduced in [15] as a way to implement, the reactive power defined in [16] as Budeanu reactive powerin a electronic system and with a reasonable cost:

$$
Q=\sum_{n=1}^{\infty} U_{n} I_{n} \sin \left(\theta_{u_{n}}-\theta_{i_{n}}\right),
$$

where $U_{n}$ and $I_{n}$ are the voltage and current phasor - RMS values of the $n$-order harmonic-, while $\theta_{u_{n}}$ and $\theta_{i_{n}}$ are their phases respectively.

The problem of applying directly this IEEE definition is that it requires the FFT application to the instantaneous values of the three-phase voltages and currents, and the calculation of the reactive power for each one of their harmonics. This is followed by their sum to obtain the reactive power per phase 
and, finally, adding the values obtained for the three phases to reach the three-phase reactive power.

In [15], a quite approximated value for the IEEE reactive power definition, (1), could be obtained if a phase lag of $\pi / 2$ to the fundamental frequency is applied, and the following mathematical operation is performed:

$$
Q=\frac{1}{T} \int_{0}^{T} u(t) i(t+T / 4) d t
$$

However, this methodology presents the disadvantage that errors are introduced if the grid frequency changes as a quarter of a cycle does not represent the same number of samples. Therefore, there exists a modification of the first method to compensate this problem that consists on adding a low-pass filter with a $20 \mathrm{db} /$ decade attenuation that improves the results obtained.

In our study the method implemented does not use the low-pass filter. It should be mentioned, under the light of the results presented in [15], that this definition should give average larger errors than those obtained using the traditional sinusoidal definition of the reactive power, [17].

\section{B. D2 definition}

This is the classical single-phase definition of reactive power, [17], that defines it from pure sinusoidal voltage and current waveforms. If $u(t)$ and $i(t)$ are the instantaneous voltage and current phase measurements, and with phasorial representation $\bar{U}=U \angle \theta_{u}$ and $\bar{I}=I \angle \theta_{i}$, then the reactive power per phase is obtained by the following expression:

$$
Q(t)=U \cdot I \sin \left(\theta_{u}-\theta_{i}\right) .
$$

In general, the fundamental frequency from pre-voltage dip measurements must be calculated to obtain the reactive power from non-sinusoidal measurements by using this value to compute the FFT, and finally obtaining the fundamental component of the current and voltage phasors and the single-phase reactive power, (3).

Applying the FFT to measured data is a well-known process, althougth obtaining the fundamental frequency with an adequate resolution could be a difficult task if only a small time interval of pre-dip voltages data is available. In our case, only one second of voltages before the generated dip was stored and, therefore, different methods have been applied in order to measure the frequency with an adequate resolution:

- The first method is based on obtaining the frequency by measuring the zero cross of the periodical waveforms. The main drawback is that, if the waveform has a high harmonic content, then the obtained frequency will not be precise enough and therefore the voltage, rather than the current, is used due to its lower harmonic content. Another difficulty arises when the sample frequency is not high enough, leading to uncertainty in the zero cross determination, as happens in the present case.

- A second method applies a frequency analysis to the measured voltage data. In this case, if the measured interval before the voltage dip is reduced, around one second as in this case, the resolution of the calculated frequency is of $1 \mathrm{~Hz}$, which is not accurate enough.

- A third alternative method that estimates the frequency from the angular speed of the voltage space vector, $\bar{V}(t)$ has also been applied. The main advantage of this method is that the frequency is obtained by means of the continuous component of the computed speed and, conseuquently, the problem of low resolution is avoided. Applying this method to the available prefault measurements of the phase voltages before the voltage dip - a one second interval-, the computed frequency is $50 \mathrm{~Hz}$ with a resolution of $\pm 0.03 \mathrm{~Hz}$.

\section{D3 definition}

This is a well-known definition introduced by Akagi in [18], where $\alpha$ and $\beta$ components of the voltage are defined as the following for a three-phase system without homopolar component $\left(v_{a}+v_{b}+v_{c}=0\right)$ :

$$
\left[\begin{array}{l}
v_{\alpha} \\
v_{\beta}
\end{array}\right]=\sqrt{\frac{2}{3}}\left[\begin{array}{ccc}
1 & -\frac{1}{2} & -\frac{1}{2} \\
0 & \frac{\sqrt{3}}{2} & -\frac{\sqrt{3}}{2}
\end{array}\right]\left[\begin{array}{l}
v_{a} \\
v_{b} \\
v_{c}
\end{array}\right]
$$

This definition can also be applied to the measured currents and, from the phase voltage and current $\alpha$ and $\beta$ components, the instantaneous power is obtained by:

$$
p(t)=v_{\alpha} i_{\alpha}+v_{\beta} i_{\beta},
$$

and the instantaneous reactive power is defined as:

$$
q(t)=v_{\alpha} i_{\beta}-v_{\beta} i_{\alpha},
$$

where $q(t)$ has a sign convention opposite to the traditional sinusoidal definition. In order to compare them, $q(t)$ must be multiplied by -1 , [19].

Other authors suggest alternative expressions for the instantaneous active and reactive power. For example, multiplying by the constant $3 / 2$ the scalar product of the space vectors $\bar{v}(t)$ and $\bar{i}(t)$, the instantaneous power can be obtained, while $3 / 2$ of the vector product will give the instantaneous reactive power, [20]. In general, the different expressions depend on the selected reference system used to project the space vectors. A simple expression to obtain the instantaneous reactive power directly from the instantaneous voltages and currents is:

$$
q(t)=\frac{1}{\sqrt{3}}\left(\left(v_{a}-v_{b}\right) i_{c}+\left(v_{b}-v_{c}\right) i_{a}+\left(v_{c}-v_{a}\right) i_{b}\right),
$$

which can be easily checked expressing the instantaneous reactive power using the measured voltages and currents.

Finally, the main restriction of this definition is that the homopolar component of the voltages and the currents must be zero, which is not a restriction for the wind turbines.

\section{D4 definition}

This definition is referred in [16] as positive-sequence reactive power, since it only uses the positive symmetrical components to calculate the value of the reactive power.

Considering that the direct sequence component of the fundamental frequency component is the most commonly 


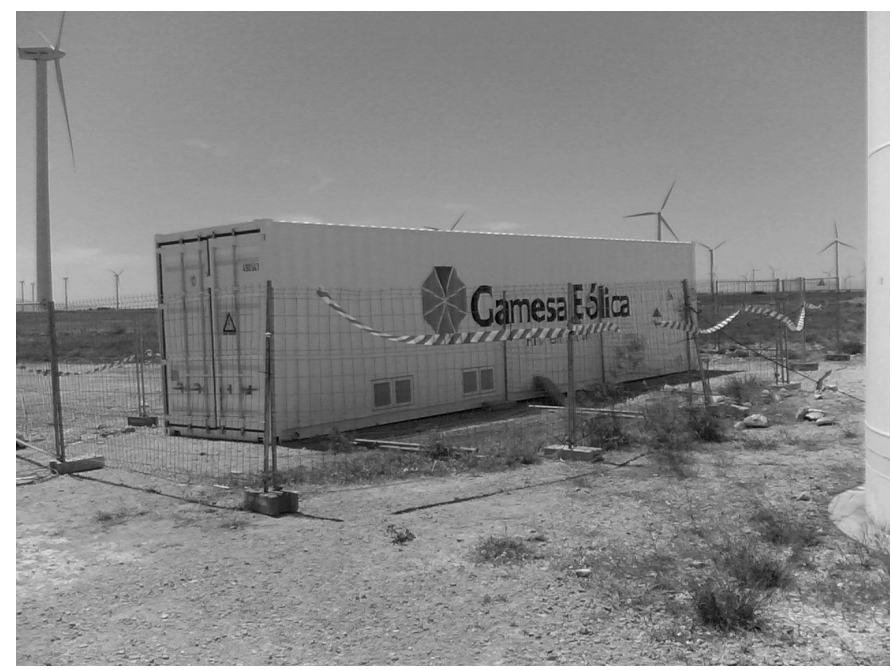

Fig. 1. Voltage dip generator system

exchanged by the electrical machines, the reactive power can be defined by calculating first the symmetrical components of the voltages and currents and then, to follow the same process as in the traditional reactive power definition section III-B).

\section{FIELD TESTS AND RESUlTS}

In the following subsections the voltage dip generator is described and the results from a three-phase voltage dip field test to a Gamesa G80 wind turbine is presented.

\section{A. Description of the voltage dip generator system}

Gamesa has designed a special installation in the medium voltage range in order to generate voltage dips and test wind turbines. This equipment can generate all kind of balanced and unbalanced faults while the network electrical conditions in the rest of the wind farm and external grid, remain unchanged. The Gamesa medium voltage dip generator is connected between the wind farm $20 \mathrm{kV}$ grid and the point of wind turbine connection, figure 1 .

The test equipment is basically composed of three elements: transformers commonly used in Gamesa wind turbines 20/0.69 kV-, medium voltage cabinets for the connections and low voltage circuit breakers for generating the different short circuits.

\section{B. Three-phase voltage dip}

In figure 2(b), the amplitude of the voltage space vector of a three-phase voltage dip and the time intervals considered by the REE grid code are represented. It represent the evolution of the line voltages during the voltage dip shown in figure 2(a) in a compact form.

As explained in subsection III-B, the continuous component of the angular speed of the complex space vector is represented in figure 2(c). It can be observed that the frquency shows its highest value around the clearance of the voltage dip.

Figure 2(d) represents the instantaneous reactive power obtained using the different definitions presented in section III. It can be observed that the obtained results are similar for the four definitions.

\section{CONCLusions}

The new grid codes require, among other magnitudes, the calculation of the reactive power generated by the wind turbines through the voltage dip, where the applied voltages can be non-sinusoidal and unbalanced. Therefore, different definitions of reactive power used in different fields of electrical engineering can be applied. Consequently, different reactive power and reactive energy computations have been be studied.

Multiple field tests have been carried out to study the behaviour of the Gamesa G80 wind turbines when they are submitted to the different types of voltage dips. To generate the voltage dips, a specially designed equipment has been used, which allows to generate all kind of balanced and unbalanced faults, required by the different national grid codes.

To take advantage of the voltage dip records -where instantaneous voltages and currents were measured in the tests, reactive power and reactive energy have been calculated according to the methodology specified by the REE grid code using different definitions. These tests have been done to a Gamesa G80 wind turbine checking that fulfils the REE grid code voltage dips requirements for existing wind turbines, not being representative its results to new ones.

A three-phase representative voltage dip test is studied. In the case of balanced three-phase faults, reactive power, reactive currents and energy computations give quite similar results for the studied reactive power definitions in the two intervals defined by the REE voltage dip grid code requirements.

\section{ACKNOWLEDGMENT}

The authors would like to thank "Gamesa" for the technical support, and the "Gamesa" people involved in all the testing campaign, and "Junta de Comunidades de Castilla-La Mancha" (PCI-05-024) for the financial support.

\section{REFERENCES}

[1] N. Jenkins, "Embedded generation," Power Engineering Journal, vol. 9, no. 3, pp. 145-150, 1995.

[2] R. C. Dugan and T. E. McDermott, "Distributed generation," IEEE Industry Applications Magazine, vol. 8, no. 2, pp. 19-25, March-April 2002.

[3] M. Bayegan, "A vision of the future grid," IEEE Power Engineering Review, vol. 21, no. 12, December 2001.

[4] M. Bollen, Understanding power quality Voltage sags and interruptions. New York: IEEE Press, 2000.

[5] I. Erlich and U. Bachmann, "Grid code requirements concerning connection and operation of wind turbines in germany," in IEEE Power Engineering Society General Meeting, San Francisco, California, June 2005.

[6] C. Abbey and G. Joos, "Effect of low voltage ride through (lvrt) characteristic on voltage stability," in Power Engineering Society General Meeting, June 2005, pp. 1901-1907.

[7] M. H. J. Bollen, G. Olguin, and M. Martins, "Voltage dips at the terminals of wind power installations," Wind Energy, vol. 8, pp. 307318, 2005.

[8] "Recommended practices and requirements for harmonic control in electrical power systems," IEEE Standard 519-1992.

[9] J. V. Milanovic and M. T. Aung, "The influence of transformer winding connections on the propagation of voltage sags," IEEE Transactions on Power Delivery, vol. 21, no. 1, January 2006.

[10] R. C. Leborgne and D. Karlsson, "Phasor based voltage sag monitoring and characterisation," in 18 International Conference on Electricity Distribution CIRED, Turin, June 2005. 


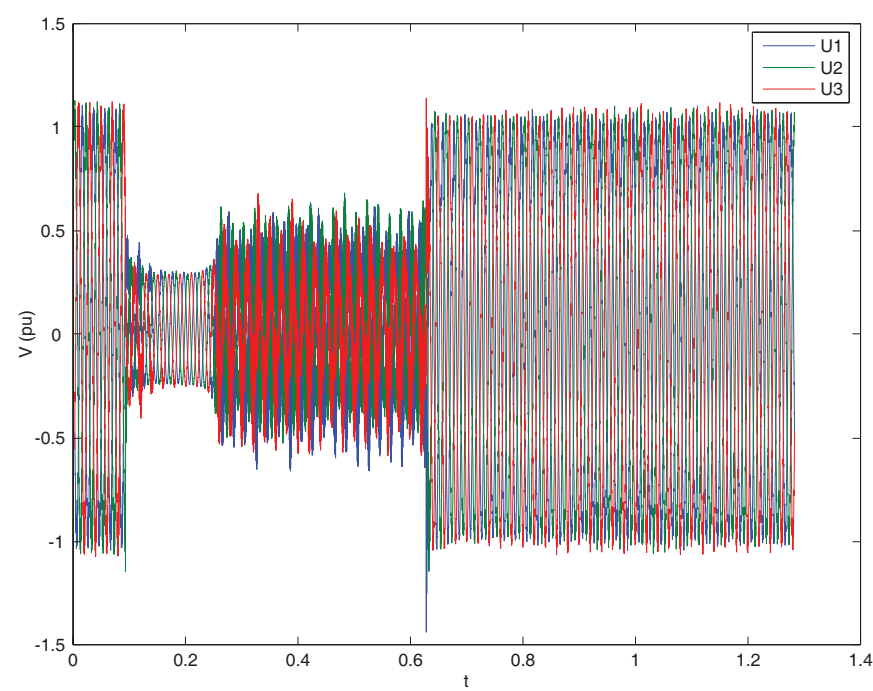

(a) Line Voltages

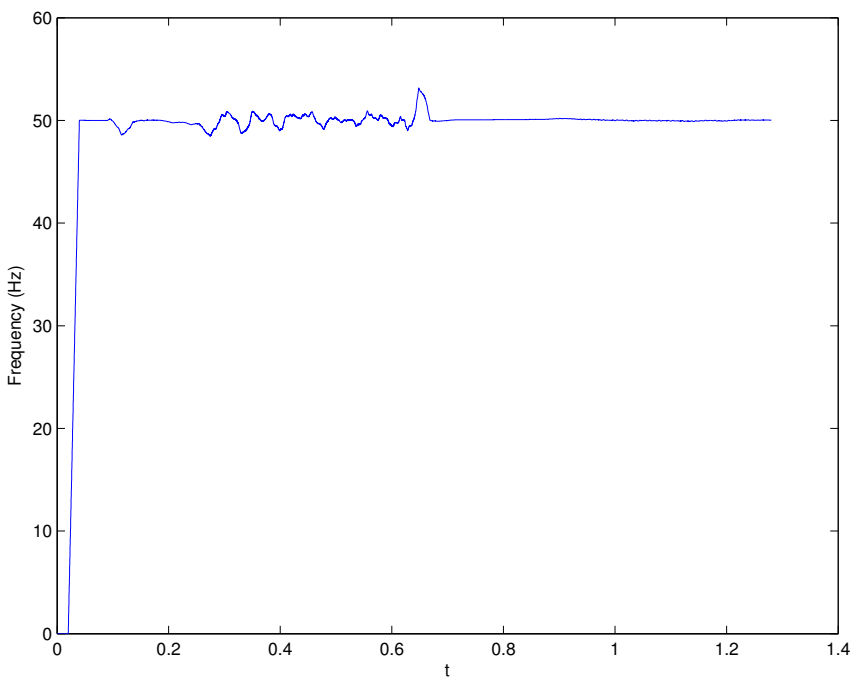

(c) Filtered frequency

Fig. 2. Three-phase voltage dip test

[11] M. H. J. Bollen, G. Olguin, and M. Martins, "Voltage dips at the terminals of wind power installations," in Nordic wind power conference, Goteborg, March 2004.

[12] G. Saccomando, J. Svensson, and A. Sannino, "Improving voltage disturbance rejection for variable-speed wind turbines," IEEE Transactions on Energy Conversion, vol. 17, no. 3, September 2002.

[13] R. Ottersten, A. Petersson, and K. Pietilinen, "Voltage sag response of pwm rectifiers for variable-speed wind turbines," Nordic Workshop on Power and Industrial Electronics, 2004.

[14] Reducing the effect of sags and momentary interruptions: a totalowning cost prospective, vol. 2, Orlando, FL, USA, 2000.

[15] E. Moulin, "Measuring reactive power in energy meters," Metering International, vol. 1, pp. 52-54, 2002.

[16] "IEEE trial-use standard definitions for the measurement of electric power quantities under sinusoidal, nonsinusoidal, balanced, or unbalanced conditions," IEEE Std 1349-2000.

[17] "International electrotechnical vocabulary," IEC 60050-131 2002.

[18] Y. K. H. Akagi and A. Nabae, "Instantaneous reactive power compensators comprising switching devices without energy storage components," Metering International, vol. IA-20, pp. 625-631, 1984.

[19] H. A. H. Watanabe, M. Aredes, "The p-q theory for active filter control: some problems and solutions," Revista Controle \& Automao, vol. 15, no. 1 , pp. 78-84, 2004.

[20] C. Schauder and H. Mehta, "Vector analysis and control of advanced

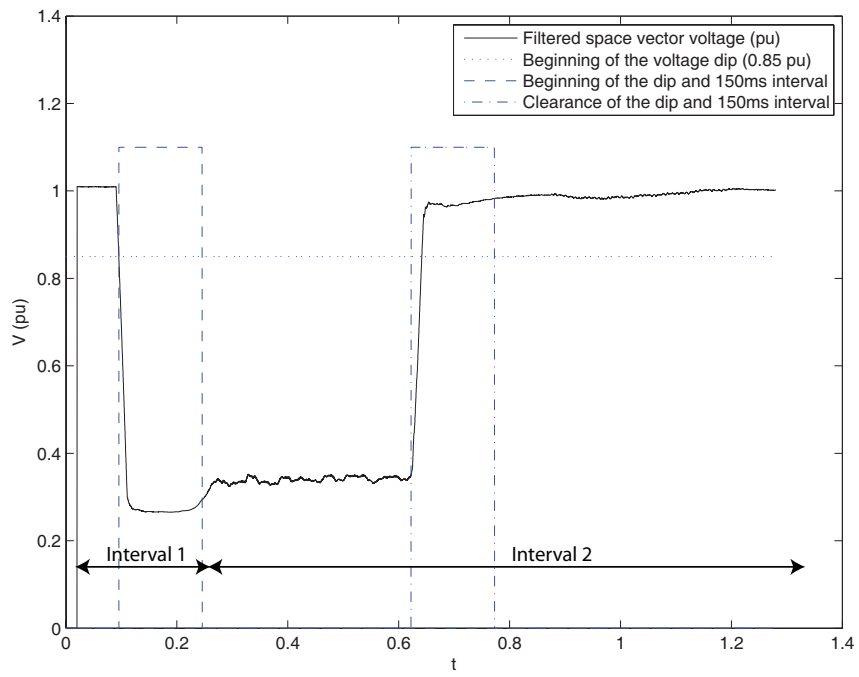

(b) Voltage space vector

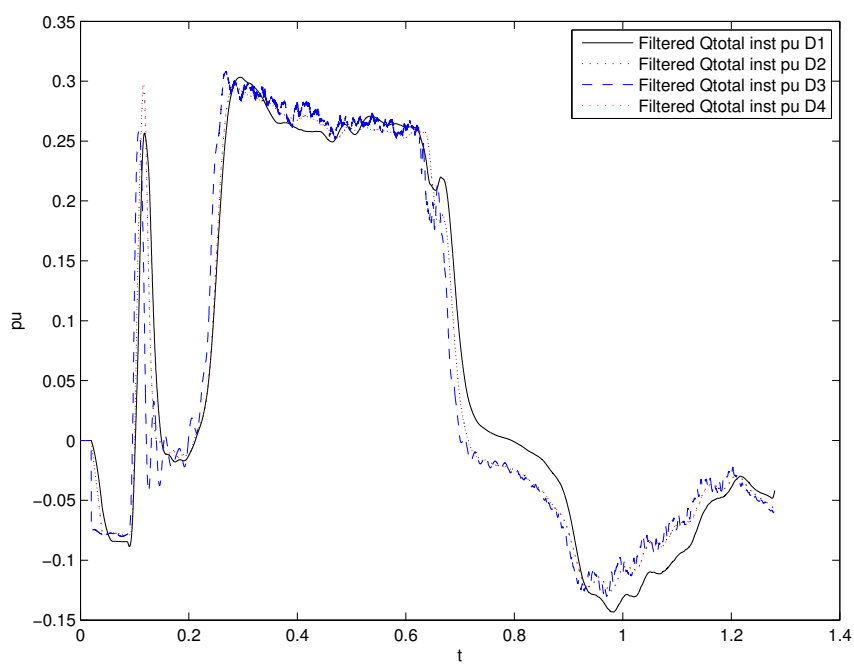

(d) Filtered generated reactive power

static var compensators," IEE Proceedings-C, vol. 140, no. 4, pp. 299 306, July 1993. 